\title{
Sistema de Procesamiento Digital de Imágenes Satelitales para Cálculo de Areas de Interés.
}

\section{System of Digital Processing of Satellite Images for Calculation of Interest} Areas.

José Luis Tinajero León. ${ }^{1}$, Pablo Eduardo Lozada Yánez. ${ }^{2}$, Monica Andrea Zabala Haro. ${ }^{3}$, Cristhy Nataly Jiménez Granizo ${ }^{4}$.

Recibido: 02-07-2019 / Revisado: 16-07-2019 /Aceptado: 05-08-2019/ Publicado: 10-09-2019

\begin{abstract}
.
DOI: https://doi.org/10.32/cienciadigital.v3i1.947

This Currently, there are several software that allow digital image processing, its deficiency lies in the high costs of its license. This article proposes the Digital Processing of Satellite Images through an analysis based on artificial vision techniques through the development of a GUIDE in Matlab (MATrix LABoratory) in which various operations are performed on a satellite image, the original image is decomposed into its various color matrices, the developed application allows the selection of the area of interest in an interactive way for easy preprocessing of the image in which the application of logical operations and filling of contours is considered, in addition the image segmentation is carried out including automatic processes and manuals of the Matlab tool, a final stage shows the approximate value of the study area prior to the processing formed by the filtering and edge detection stage. The results allowed to determine that while the Otsu method does not discriminate isolated areas of the image
\end{abstract}

\footnotetext{
${ }^{1}$ Escuela Superior Politécnica de Chimborazo, Facultad de Informática y Electrónica. Riobamba, Ecuador. joseluis.tinajero@espoch.edu.ec

${ }^{2}$ Escuela Superior Politécnica de Chimborazo, Facultad de Informática y Electrónica. Riobamba, Ecuador. plozada@espoch.edu.ec

${ }^{3}$ Escuela Superior Politécnica de Chimborazo, Facultad de Informática y Electrónica. Riobamba, Ecuador. monica.zabala@espoch.edu.ec

${ }^{4}$ Universidad Nacional de Chimborazo, Facultad de Ciencias de la Educación y Tecnologías. Riobamba, Ecuador.ccjimenez@unach.edu.ec
} 
and considers them as part of the area of interest on which the calculation of the area will be made, the manual tuning process to find the threshold of the image discriminates those isolated areas that do not belong to the area improving the calculation of the area of interest.

Keywords: Satellite, Satelital Image, Digital Image Processing, GUIDE, Matlab.

\section{Resumen.}

En la actualidad existen varios softwares que permiten realizar procesamiento digital de imágenes su deficiencia radica en los elevados costos de su licencia. El presente artículo plantea el Procesamiento Digital de Imágenes Satelitales mediante un análisis basado en técnicas de visión artificial mediante el desarrollo de un GUIDE en Matlab en el cual se realizan diversas operaciones sobre una imagen satelital, la imagen original se descompone en sus diversas matrices de color, la aplicación desarrollada permite la selección del área de interés de forma interactiva para facilidad del preprocesamiento de la imagen en el cual se considera la aplicación de operaciones lógicas y relleno de contornos, de manera adicional se realiza la segmentación de la imagen incluyendo procesos automáticos y manuales propios de la herramienta Matlab, una etapa final muestra el valor aproximado del área de estudio previo al procesamiento conformado por la etapa de filtrado y detección de los bordes. Los resultados permitieron determinar que mientras el método Otsu no discrimina zonas aisladas de la imagen y las considera como parte de la zona de interés sobre la cual se realizará el cálculo del área, el proceso de tunning manual para hallar el umbral de la imagen discrimina aquellas zonas aisladas que no pertenecen al área mejorando el cálculo del área de interés.

Palabras claves: Satélite, Imagen Satelital, Procesamiento Digital de Imágenes, GUIDE, Matlab.

\section{Introducción.}

El procesamiento digital de imágenes ha experimentado un crecimiento considerable en los últimos años, pues se pretende emular ciertas capacidades que poseen los seres vivos, se realiza la digitalización del mundo real mediante dispositivos electrónicos para obtener capturas de un determinado espacio, dichas capturas son procesadas mediante Software 
específicos que permiten realizar diferentes métodos de análisis de los espacios estudiados.

Para el análisis de áreas específicas se procesa en la mayoría de los casos con software específicos bajo ciertas limitaciones en la versión gratuita y licenciada, ante estas dificultades se propone una alternativa al procesamiento de imágenes para análisis de casos específicos que incluye el post procesamiento de la imagen satelital con la ayuda de algoritmos de procesamiento, los resultados se presentan al usuario en forma intuitiva a través de una interfaz gráfica que permite seleccionar el área de interés, segmentar la imagen original mediante métodos manuales y automáticos, binarización de las imágenes.

El artículo presenta los conceptos teóricos en los cuales se fundamenta la investigación, además se muestra el análisis de los resultados en base al procesamiento digital de imágenes realizado en el GUIDE de Matlab, se realiza también una breve discusión de los resultados obtenidos, y finalmente, las conclusiones establecidas.

\section{Metodología.}

Se desarrolla la implementación de un sistema de procesamiento digital de imágenes satelitales las acciones de descomposición de matrices de la imagen original, selección del área de interés para discriminar elementos no relevantes al caso de estudio para finalmente crear una nueva imagen binaria del objeto de forma manual y también automática para así ejecutar su post procesamiento y ejecutar cálculos de interés.

\section{Infraestructura Satelital}

De acuerdo a Andy (2018), existe 4857 satélites actualmente orbitando el planeta, en 2018 United Nations Office for Outer Space Affairs (UNOOSA) ha registrado 204 objetos lanzados hacia el espacio (Andy, 2018). En 2017 el número de objetos lanzados al espacio alcanzó la cantidad de 453, representando el 5\% de los objetos lanzados al espacio. Según UNOOSA (2016), puntualiza que en la historia se han lanzado 8126 objetos al espacio, y más del 22\% de estos se encuentran solo en los últimos ocho años (Affairs, 2016).

La mayoría de estos objetos son cubesat y entre los principales objetivos incluye servicios de comunicación, misiones para observación de la tierra contando con 661 satélites aproximadamente y para el servicio de navegación por satélites las constelaciones que están 
bajo desarrollo han alcanzado ha incrementado a 121 satélites un $12 \%$ más que el año pasado, entre otras.

\section{Sistemas Satelitales para la observación de la Tierra}

La observación de la tierra representa una de las principales actividades de los vehículos espaciales ya que a través de la información satelital es posible monitorear los recursos y fenómenos naturales categorizándolos dentro programas específicos que permiten monitorear actividades que pueden afectar a los humanos. Los sensores a bordo permiten la recolección de información sobre el área de interés bajo diferentes formatos, las disponibles actualmente corresponden a las imágenes ópticas consideradas las de mayor interés por los beneficios que muestra en el estudio de casos específicos, así también, las imágenes de radar, imagen infrarroja utilizadas para servicios de monitoreo de cambios climáticos terrestres y espaciales, recursos hídricos, verificación de desastres naturales y monitoreo remoto, entre otros. El esquema de obtención, procesamiento y acceso a la información está estructurado en niveles como se ve en la Fig. 1 y Fig. 2 (EESA, 2019).

Figura 1. Esquema general de la infraestrcutural satelital

\section{Esquema General}

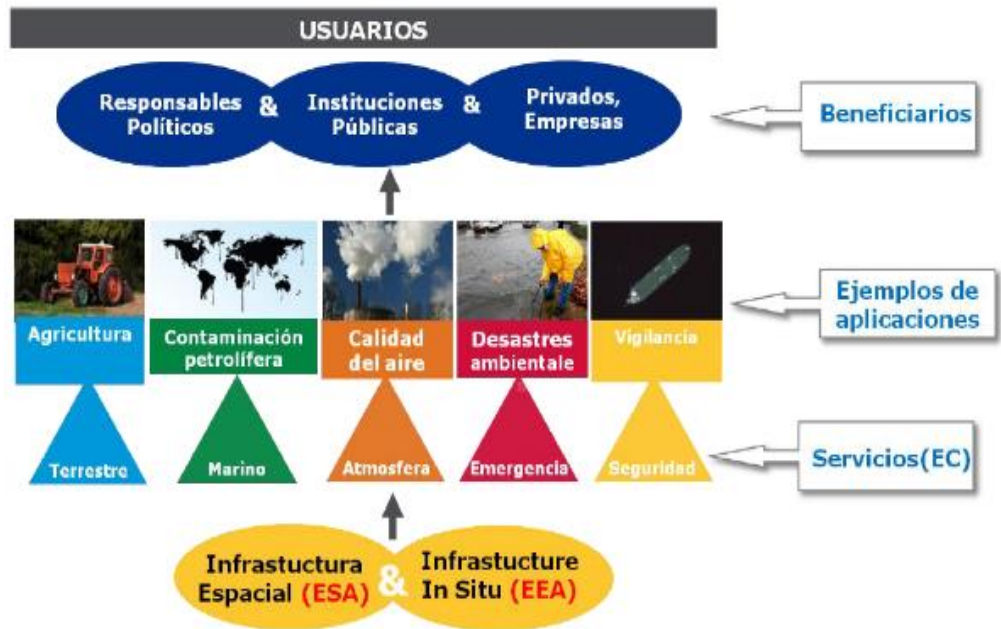

DATOS DE TELEDETECCIÓN

Fuente: http://www.esa.int/copernicus 
Figura 2. Arquitectura de sistemas satelital de observación de la tierra

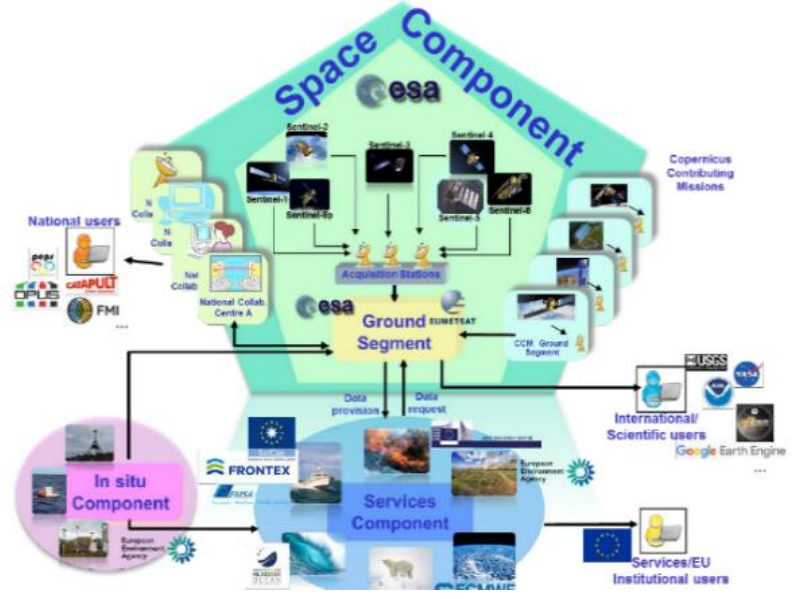

Fuente: http://www.esa.int/copernicus

El dato bruto o información original obtenido por cada vehículo satelital a través de los sensores es procesada de acorde las aplicaciones y servicios finales a brindar a los usuarios bajo las normativas de cada organización que está a cargo de su procesamiento y distribución en forma libre o de pago realizada con software específicos para su posterior publicación a través de sitios oficiales web.

Nuevos países se han integrado a la carrera espacial con diversos proyectos en diferentes áreas, también ha sido de interés el participar en proyectos que integren la observación de la tierra sin embargo por cuestiones políticas y económicas su participación es reducida. Actualmente los países que lideran la administración y lanzamiento de satélites de observación de tierra (EO) y según United Nations (2018), E.E.U.U. lidera el control de más de la mitad de los satélites EO, después de EE. UU está China con un 14,4\%, y luego India, Japón y Rusia, que tienen cada uno más del 3\%. el Reino Unido solo figura como controlador en 4 satélites, gestionados por sus agencias espaciales que incluye a la Agencia Espacial Europea (ESA) y la Administración Nacional de Aeronáutica y Espacio (NASA) (Nations, 2018).

\section{Programa de observación de la Tierra. Copernicus}

En el programa de observación de la tierra, ESA reúne su flota de satélites y los agrupa en diferentes misiones distribuidas como se observa en la Fig. 3, y englobadas bajo el programa denominado el cual tiene como objetivo el monitoreo del ambiente, mitigación de los efectos 
del cambio climático y seguridad civil en colaboración con la industria, compañías privadas, Agencias espaciales nacionales e internacionales y Organización Europea para la Explotación de Satélites Meteorológicos (EUMETSAT) (Copernicus, 2018).

Figura 3. Misiones de Observación de la Tierra controladas por la ESA

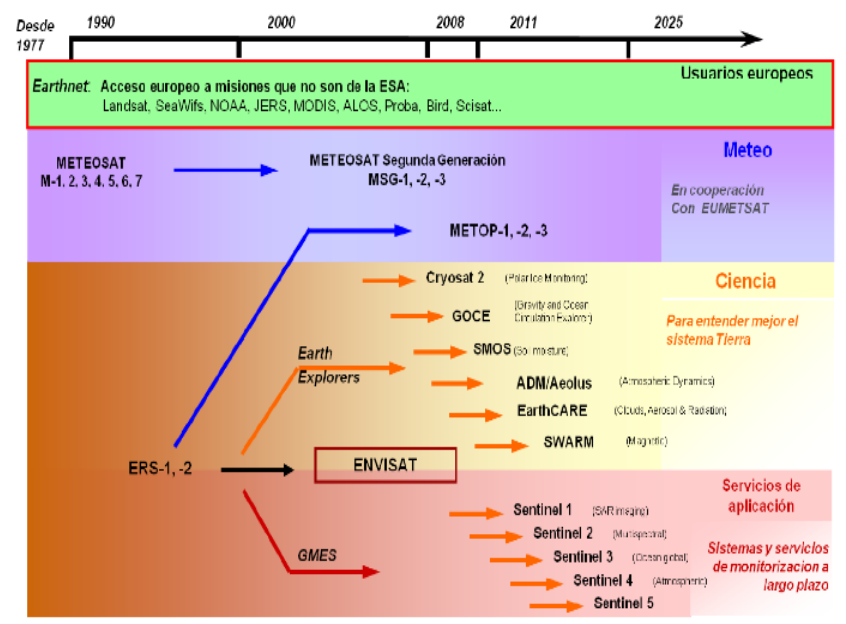

Fuente: https://www.copernicus.eu/en

Misiones como Soil Moisture and Ocean Salinity (SMOS) responsable de medir la humedad del suelo y salinidad oceánica, SWARM responsable de medir el campo geomagnético de la tierra y sus variaciones temporales en el interior de la tierra junto con el clima (González, 2017). Conceptos y áreas temáticas propuestas con alta prioridad se considera el monitoreo del $\mathrm{CO} 2$, ambiente marino y observaciones polares, monitoreo de la tierra por imágenes térmicas y desastres naturales, uno de los productos finales se observa en la Fig. 4 (Ruescas, 2009).

Figura 4. Campo térmico estival representativo del intenso calentamiento en la capa superficial del mar con entrada de aguas frescas atlánticas en el mar de Alborán cuyo hundimiento provoca ascensos de aguas frías del fondo (Imagen NOAA HRPT, 13/07/2004)

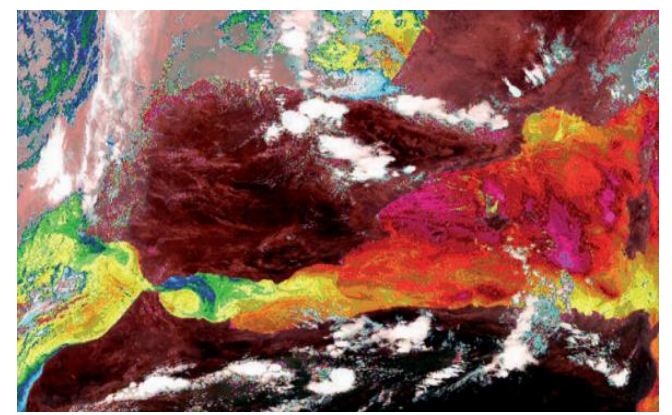

Fuente:https://www.researchgate.net/publication/241775231 La formacion del campo te rmico marino en la cuenca occidental del Mediterraneo 


\section{Plataforma de acceso Web}

Los datos brutos son preparados por los centros procesamiento masivo de la ESA para su posterior publicación bajo la normativa de libre acceso bajo ciertas restricciones de calidad y disponibilidad de cobertura. En forma general, el sitio web la interfaz permite buscar y filtrar resultados por fechas, satélites y área de interés. Sin embargo, hay que considerar que la disponibilidad de las imágenes solo se limita a la cobertura espacial y temporal del satélite además de la calidad por defecto brindada por la plataforma.

En orden de acceder a las imágenes es necesario el registro en la plataforma para su posterior descarga. Hay que considerar ciertas restricciones de cobertura pues la huella satelital como se observa en la Fig. 5 Eumesat (2018), define que es posible que no coincida con los criterios de interés de estudio, así mismo la calidad y escala no permite identificar detalles importantes que permitan discriminar criterios en la observación de fenómenos naturales (EUMETSAT, 2018).

Figura 5. Plataforma web Copernicus

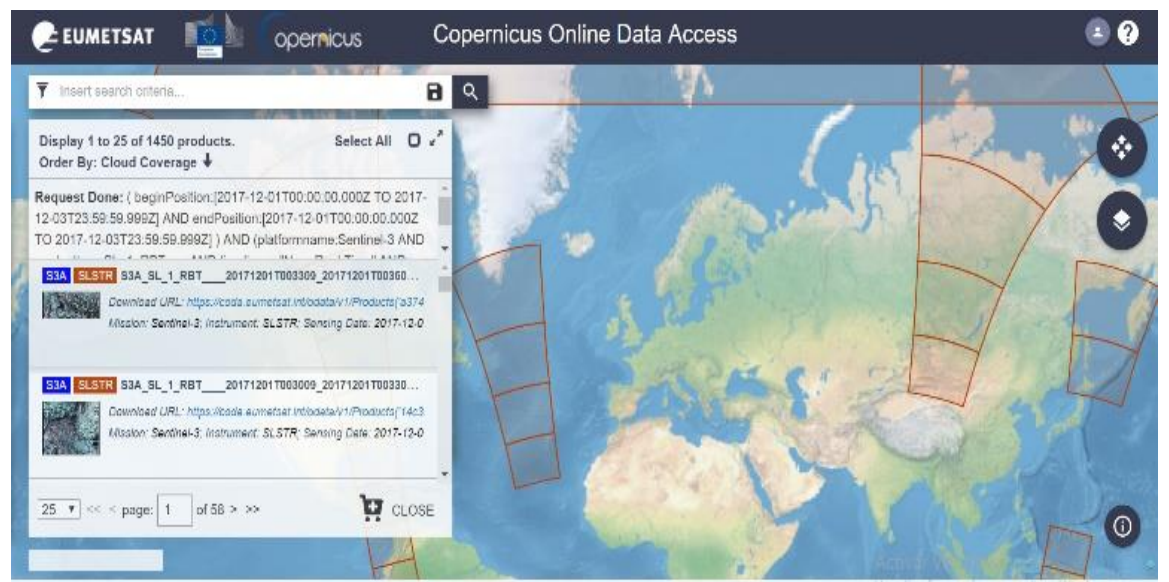

Fuente:https://www.eumetsat.int/website/home/Data/DataDelivery/CopernicusOnlineDataA ccess/index.html

\section{GUIDE Matlab}

Es un entorno de programación visual disponible en MATLAB para realizar y ejecutar programas que necesitan un ingreso de datos de manera continua, posee las características básicas de los programas de visuales. (Barragán, 2017).

Un buen GUIDE puede permitir que los programas sean más fáciles de usar al proporcionarles una apariencia consistente y con controles intuitivos como botones, 
pulsadores, cuadros de lista y demás elementos existentes para desarrollar una interfaz gráfica amigable.

\section{Visión Artificial}

La visión artificial tiene como objetivo extraer información del mundo físico a partir de imágenes, utilizando un ordenador, se consideran tres niveles de procesamiento:

- Nivel Bajo considera el proceso de captura de la imagen y preprocesamiento que tiene por finalidad reducir el ruido, realzar el contraste o características.

- Nivel Medio está conformado por la segmentación, descripción y reconocimiento de características propias de la imagen.

- Nivel Alto se caracteriza por la interpretación de los objetos encontrados en la captura.

\section{Segmentación}

Es el proceso por el cual se divide a una imagen en regiones homogéneas que poseen una o varias características en común facilitando el posterior procesamiento digital de las imágenes, el proceso de segmentación está definido por la umbralización que puede ser realizada por métodos manuales o automáticos los mismo que consisten en convertir una imagen en escala de grises a una imagen binaria de manera que los objetos y elementos en estudio se puedan definir con un valor diferente al resto de los pixel que componen la captura.

\section{Filtrado Espacial}

Es una técnica que permite mejorar o modificar la imagen a ser procesada, en la cual se trata de resaltar o atenuar algunas características que necesitan ser analizadas dentro de las capturas realizadas.

\section{Detección de Bordes}

Se emplea un filtro pasa alto, cuya finalidad es resaltar o preservar los contornos de un elemento específico dentro de la captura realizada, considerando el valor de los pixeles analizados y el de sus vecinos (García, 2018).

\section{Metodología}

Se desarrolla la implementación de un sistema de procesamiento digital de imágenes satelitales las acciones de descomposición de matrices de la imagen original, selección del 
área de interés para discriminar elementos no relevantes al caso de estudio para finalmente crear una nueva imagen binaria del objeto de forma manual y automática para así ejecutar su post procesamiento y ejecutar cálculos de interés. Para el caso se presenta el análisis de varias imágenes satelitales que están conformadas por recursos hídricos.

\section{Descomposición de Matrices}

El proceso de descomposición en diversas matrices de color es destacar elementos de posible análisis dentro de la imagen descargada, tal como se muestra en la Fig.6.

Figura 6. Descomposición de la Imagen Original en Matrices de Color
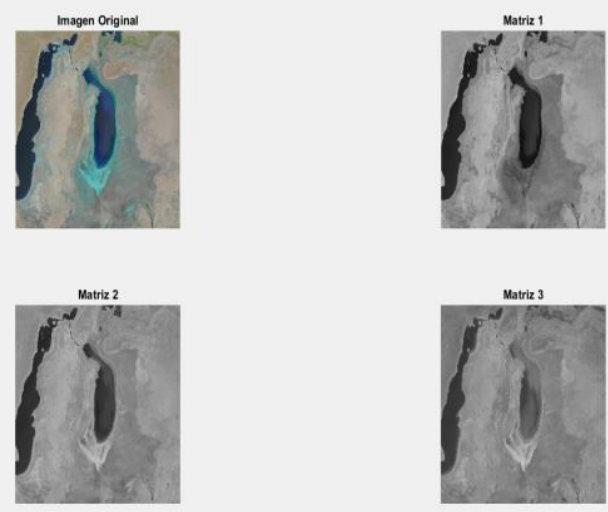

Fuente: https://es.slideshare.net/FLliuya/introduccion-al-procesamiento-de-imagenes-con$\underline{\text { matlab }}$

En la Fig. 6 se visualiza la imagen satelital representada en sus diversas matrices de color, para la siguiente etapa de análisis se considera la matriz 1en la cual se visualiza de forma clara los detalles de color azul de la captura a ser procesada (Manna, 2016).

\section{Selección del Área de Interés}

El área de interés representa una sección específica a la que se expone al análisis en detalle, la interfaz gráfica permite la selección del objeto a través del cursor similar a las características de las plataformas web.

En la Fig. 7 se muestra el proceso realizado, para el caso de estudio seleccionado se analiza el lago que se encuentra presente en el centro de la captura. 
Figura 7. Selección-Recorte del Área de Interés
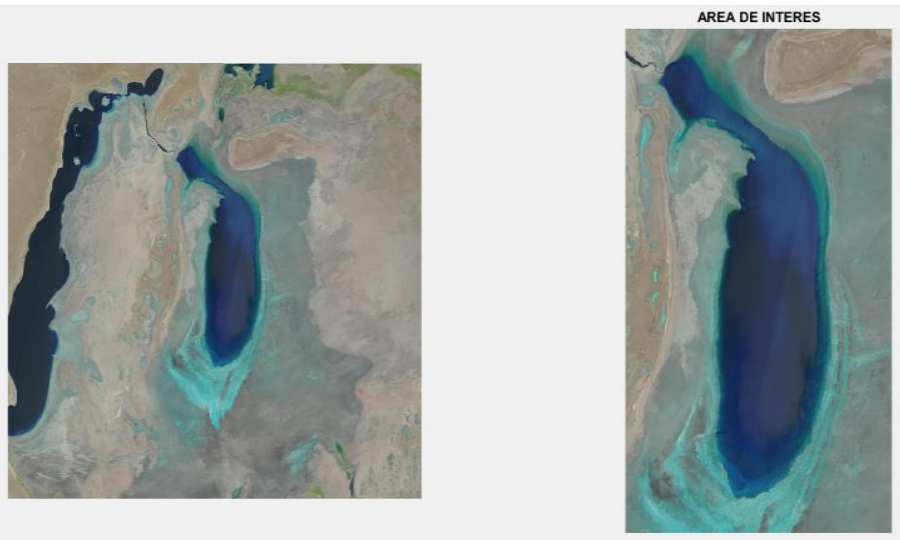

Fuente: https://es.slideshare.net/FLliuya/introduccion-al-procesamiento-de-imagenes-con$\underline{\text { matlab }}$

\section{Segmentación de la imagen}

El objetivo de estudio es calcular la posible área conformada por el elemento de interés para realizar estudios de afectación climática sobre diversas capturas realizadas a diversos recursos hídricos. Se procede a segmentar la imagen de dos formas, la primera corresponde al método automático, aplicando el método Otsu, considerado uno de los mejores métodos de selección de umbral pues no necesita supervisión humana ni previa información de la imagen antes de su procesamiento, posee una desventaja y respecto del tiempo que emplea para seleccionar el umbral óptimo, para la imagen analizada el valor óptimo de umbral es 0.329412 , la Fig. 8 muestra la imagen binaria obtenida con el valor calculado por el método automático (Díaz, 2016).

Figura 8. Segmentación Basada en el Método Otsu.

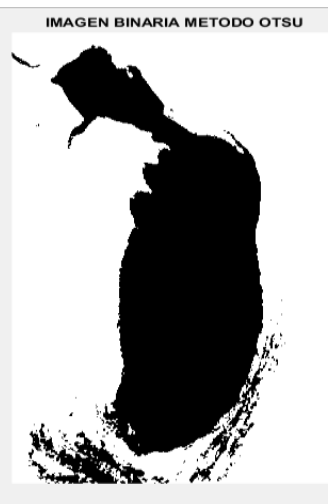

Fuente:http://www.iiis.org/CDs2016/CD2016Summer/papers/CA040RP.pdf

Para realizar la comparación de resultados, se realiza la segmentación de la imagen 
mediante un método manual, es decir, se binariza la imagen mediante un valor establecido por el usuario, el mismo que es ingresado al GUIDE mediante una barra de deslizamiento que varía entre valores establecidos en el rango de 0 y 1 , el valor ingresado realiza la agrupación de pixeles de acuerdo a la afinidad que estos poseen, según el umbral considerado se puede identificar mayor o menor cantidad de pixeles en el elemento de estudio, en la Fig. 9 se muestra el proceso de Binarización de la imagen considerando un valor de umbralización de 0.25 (Fresno, 2012)

Figura 9. Segmentación Basada en el Método Manual.

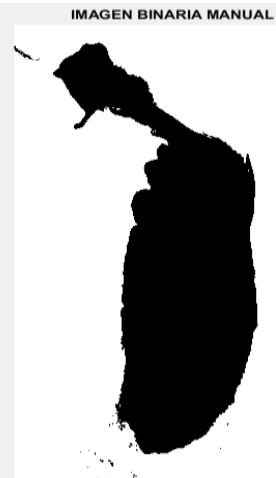

Fuente:https://pdfs.semanticscholar.org/10bd/44ffd42b93b7757ad05c35588e8598cf5633.pdf

\section{Cálculo de Área de Interés}

De acuerdo con la Fig. 8 y Fig. 9 realiza el preprocesamiento de las capturas de forma automática o manual, dependiendo de las características de las imágenes binarias permitiendo verificar la diferencia existente entre las imágenes después de su procesamiento.

Los resultados del preprocesamiento realizado con la imagen binaria basada en el método Otsu se muestra en la Fig. 10, se realiza la negación de la imagen binaria la cual consiste en negar los pixeles de la imagen para obtener el elemento de estudio en color blanco y fondo de la imagen en color negro, adicionalmente se realiza el relleno de la región de interés para eliminar cualquier pixel aislado que pueda distorsionar el procesamiento de la imagen (Madrid, 2015) 
Figura 10. Preprocesamiento de la Imagen Basada en el Método Otsu.

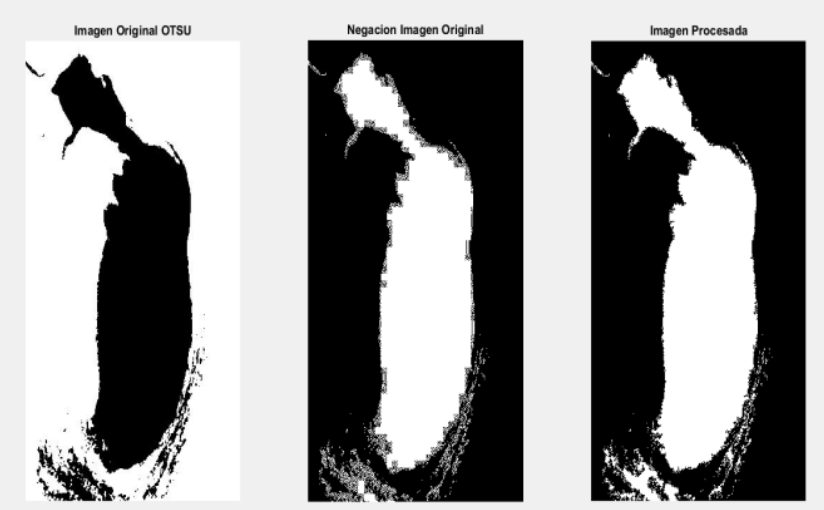

Fuente:http://www.elai.upm.es/webantigua/spain/Asignaturas/Robotica/PracticasROVA/prROVA 5Segmentacion.pdf

Al realizar el cálculo del área de interés se realiza el procesamiento de la imagen, en este procedimiento se aplica un filtro pasa bajo el cual analiza un conjunto de pixeles formado por el pixel en estudio y sus ocho vecinos, se emplea el filtro de mediana, también se extrae los bordes de la imagen filtrada obteniéndose una forma bastante clara del objeto analizado, en la Fig. 11 se muestra los resultados obtenidos:

Figura 11. Procesamiento de la Imagen con Umbral Automático.
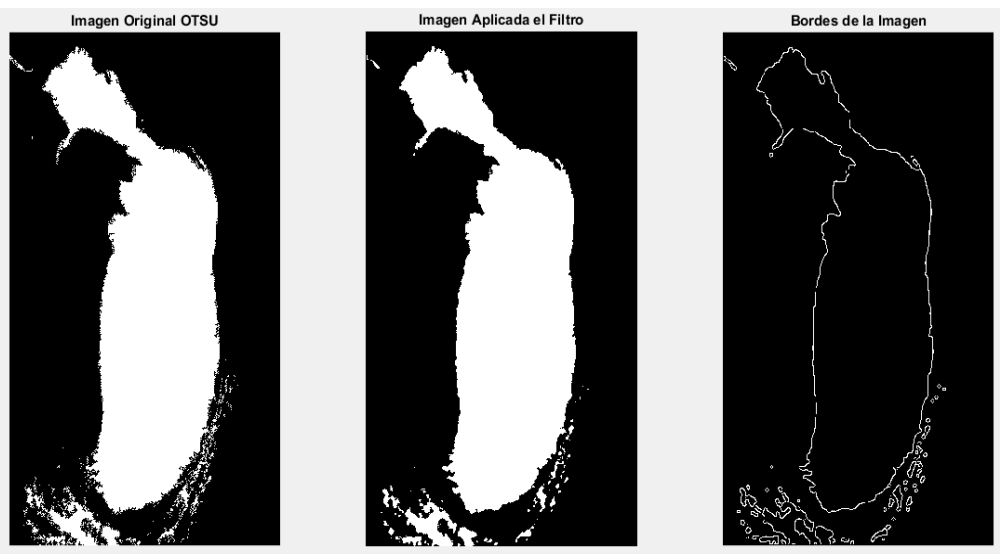

Fuente:http://www2.elo.utfsm.cl/ elo328/pdf1dpp/PDI18_Segmentacion_1dpp.pdf

Finalizado el procesamiento de las imágenes el valor se muestra en el GUIDE desarrollado considerando que el área calculada tiene unidades de medida $m^{2}$, el dato calculado se muestra en la Fig. 12 (Vara, 2016) 
Figura 12. Valor Aproximado del Área de la Imagen Automática

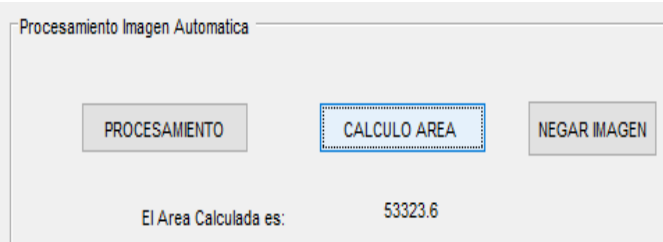

Fuente:https://www.researchgate.net/publication/305026943_Ensenando_Optica_Coherent e_usando_Matlab_GUIDE

Con la imagen binaria obtenida de forma manual se realiza un procesamiento similar al anteriormente descrito, la diferencia radica en la reducción del espacio analizado, pues en la imagen del método automático se logra eliminar varios pixeles que se asumen que no corresponden al lago, en la Fig., 13 se muestran los resultados obtenidos del preprocesamiento aplicado a la imagen binaria manual.

Figura 13. Preprocesamiento de la Imagen Manual.
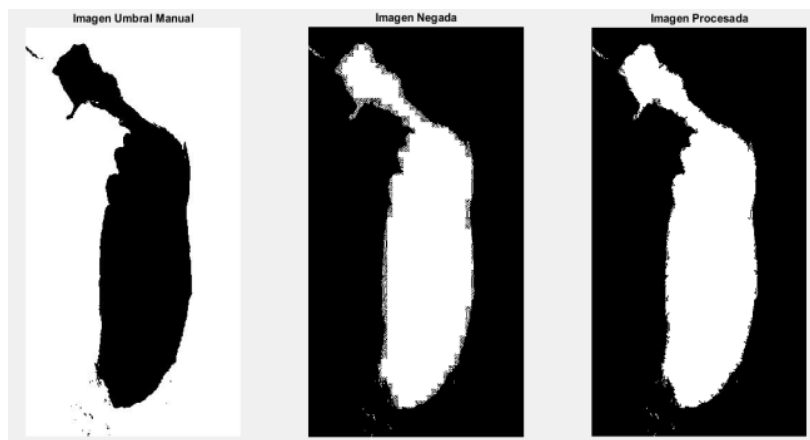

Fuente:http://www2.elo.utfsm.cl/ elo328/pdf1dpp/PDI18_Segmentacion_1dpp.pdf

En la Fig. 14 se analiza el procesamiento de la imagen binaria manual, tras ser aplicada el filtro de la mediana y la detección del borde dentro de la misma:

Figura 14. Procesamiento de la Imagen con Umbral Manual.
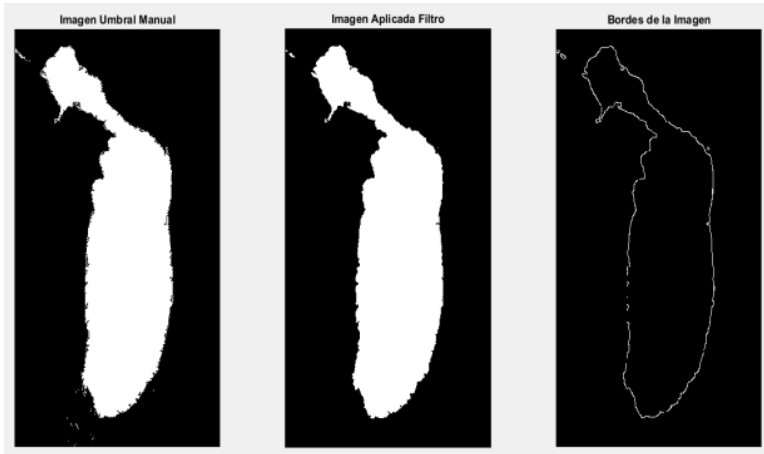

Fuente:http://www2.elo.utfsm.cl/ elo328/pdf1dpp/PDI18 Segmentacion 1dpp.pdf 
El valor aproximado que corresponde al área de estudio, por este método se muestra en la fig. 15 .

Figura 15. Valor Aproximado del Área de la Imagen Manual

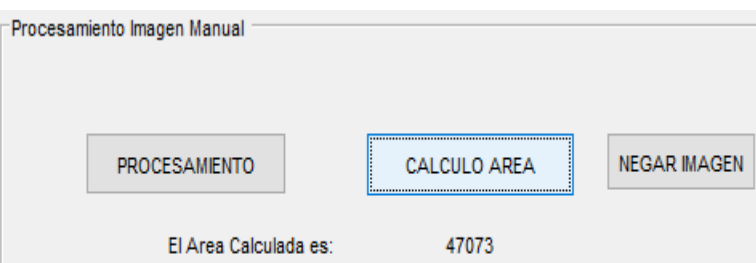

Fuente:https://www.researchgate.net/publication/305026943 Ensenando Optica Coherente u sando Matlab GUIDE

\section{Resultados}

Para el procesamiento y análisis de la imagen satelital se desarrolló un GUIDE en Matlab, en el cual se crearon varias pantallas que permiten realizar las operaciones descritas en el apartado anterior. En el entorno de desarrollo gráfico se debe ingresar el nombre de la foto descargada y de la cual se pretender realizar el cálculo del área de un elemento específico dentro de la captura.

\section{Detección de Bordes}

En la Fig. 16 se evidencia una gran diferencia en dichas imágenes, en el procesamiento de la imagen binaria manual se eliminan pixeles aislados que se encuentran en la parte inferior de la imagen, en tanto, la imagen binaria automática posee una gran cantidad de pixeles que para la apreciación del programador no forman parte del elemento en estudio.

Figura 16. Comparación de los Bordes Detectados.
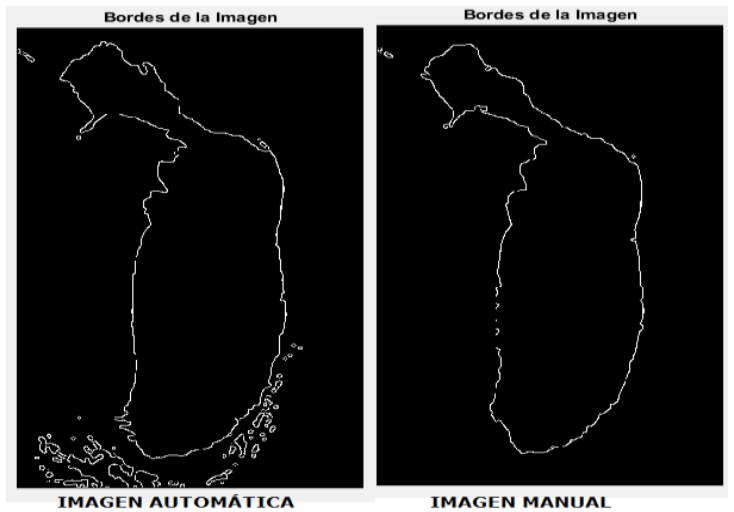

Fuente: http://www.sc.ehu.es/ccwgrrom/transparencias/pdf-vision-1-transparencias/capitulo$\underline{\text { 6.pdf }}$ 


\section{Valor de Umbralización}

Existe una gran diferencia de los valores calculados entre los diversos métodos, la variación entre dichos resultados corresponde a la cantidad de información presente en la imagen binaria, en la imagen manual se eliminan la mayor cantidad de pixeles aislados que partiendo de la imagen original no forman parte del elemento en estudio. En la Fig. 17 se muestran el área del elemento estudiado (Unipython, 2018)

Figura 17. Comparación de las imágenes binarias.
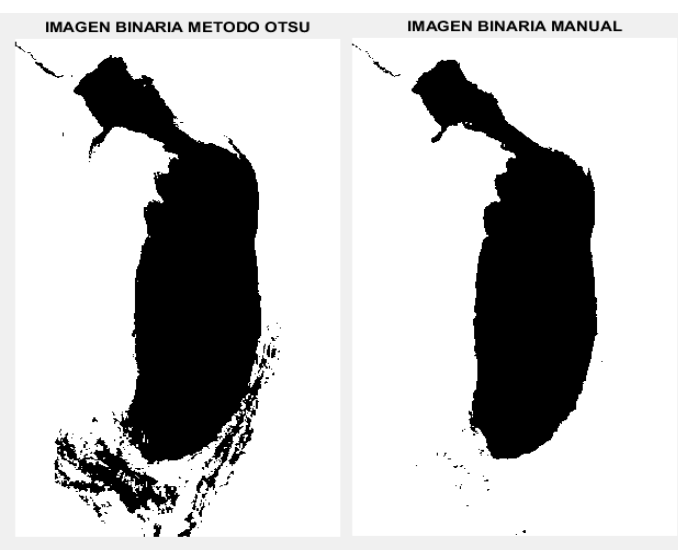

Fuente: https://unipython.com/umbralizacion-una-imagen/

\section{Cálculo del Área}

Existe una gran diferencia de los valores calculados entre los dos métodos en análisis, la variación en los datos se evidencia en lo expuesto al valor de Binarización considerado para cada uno de los procesamientos realizados a las diversas imágenes. En la Fig. 18 se muestran el área del elemento estudiado.

Figura 18. Comparación del área de las imágenes binarias.
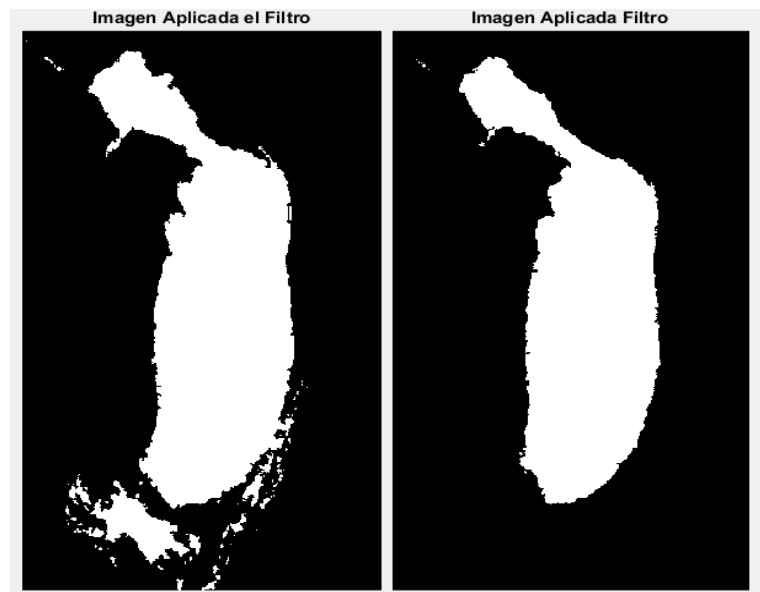

Fuente: https://unipython.com/umbralizacion-una-imagen/ 


\section{Análisis Estadístico}

Para la validación de resultados se empleó la prueba T-Student para medidas de dos muestras emparejadas considerando un nivel de confianza del 5\%, para aplicar dicha prueba se consideran valores normalizados. La tabla 1, muestra los datos empleados para el estadístico.

Tabla 1. Valores Normalizados de las Áreas

\begin{tabular}{ll}
\hline Área Automática & Área Manual \\
\hline 10,88413 & 10,75945 \\
13,49088 & 13,43656 \\
11,98616 & 11,91382 \\
10,95777 & 10,83876 \\
8,270397 & 8,238373 \\
11,16667 & 11,1962 \\
9,809413 & 9,708166 \\
10,1793 & 10,08641 \\
\hline
\end{tabular}

Fuente: T-Student Test

Los resultados estadísticos se muestran en la Tabla2.

Tabla 2. Valores Estadísticos Prueba T-Student.

\begin{tabular}{lll}
\hline Parámetro & Área Automática & Área Manual \\
\hline Media & 10,84309 & 10,77222 \\
Varianza & 2,366397 & 2,372746 \\
Observaciones & 8 & 8 \\
Coeficientes de correlación de Pearson & 0,999444 & \\
Diferencia hipotética de las medias & 0 & \\
Grados de libertad & 7 & \\
Estadístico t & 3,900947 & \\
$\mathrm{P}(\mathrm{T}<=\mathrm{t})$ una cola & 0,002946 & \\
\hline
\end{tabular}

Fuente: T-Student Test

\section{Discusión}

El trabajo desarrollado muestra la implementación de un sistema que, en base al procesamiento de imágenes satelitales, permite el determinar el área de regiones que el usuario elige de acuerdo a sus necesidades. Para realizar el procesamiento, se aplicaron varias operaciones sobre la imagen tratada: descomposición de matrices de la imagen, selección del área de interés, discriminación de elementos no relevantes al caso de estudio y la aplicación de dos métodos de binarización con el fin de calcular el área de la región seleccionada.

Con la finalidad de mejorar los resultados al calcular el valor del área de interés, se utilizaron y compararon dos métodos para encontrar el valor óptimo de umbralización que permita 
segmentar y binarizar la imagen: el método Otsu que proporciona el umbral óptimo para la segmentación de la imagen, bajo el criterio de máxima varianza entre fondo y objeto y un método manual que implementa un método de tunning para la selección del valor del umbral. Los resultados permitieron determinar que mientras el método Otsu no discrimina zonas aisladas de la imagen y las considera como parte de la zona de interés sobre la cual se realizará el cálculo del área, el proceso de tunning manual para hallar el umbral de la imagen discrimina aquellas zonas aisladas que no pertenecen al área mejorando el cálculo del área de interés. Adicionalmente, se implementó una GUIDE en Maltab que permite ver la imagen cada vez que el proceso de tunning manual modifica el valor de la imagen a procesar.

A continuación, la tabla 3, muestra el resultado de la aplicación de ambos métodos (automático y manual) sobre otras imágenes con similares características a las mostradas en las secciones anteriores. Pese a que la variación del valor del umbral entre ambos métodos está en el orden de pocas décimas, de los casos de estudio analizados, se puede concluir que el método de tunning manual permite encontrar un valor más preciso del área de interés debido a que no se consideran regiones aisladas.

Tabla 3. Valores Obtenidos de Diversas Imágenes de Lagos.

\begin{tabular}{llllll}
\hline Imagen & Descripción & $\begin{array}{l}\text { Valor } \\
\text { Otsu }\end{array}$ & $\begin{array}{l}\text { Valor } \\
\text { Manual }\end{array}$ & Área Otsu(m2) & $\begin{array}{l}\text { Área } \\
\text { Manual }(\mathrm{m} 2)\end{array}$ \\
\hline Imagen 1 & Lago Mar de Aral & 0.3294 & 0.25 & 53323.6 & 47073.0 \\
Imagen 2 & Lago Mar de Aral & 0.2863 & 0.24 & 722794.0 & 684578.0 \\
Imagen 3 & Lago Poopó & 0.4863 & 0.45 & 160517.0 & 149316.0 \\
Imagen 4 & Lago Vietma & 0.4627 & 0.36 & 57398.4 & 50958.4 \\
Imagen 5 & Lago Pellegrini & 0.3882 & 0.37 & 3906.5 & 3783.38 \\
Imagen 6 & Lago Musters-Rio Chubut & 0.3412 & 0.45 & 70733.5 & 72853.0 \\
Imagen 7 & Lago Colhue & 0.3490 & 0.27 & 18204.3 & 16451.4 \\
Imagen 8 & Lago Argentino & 0.4431 & 0.35 & 26352.1 & 24014.5 \\
\hline
\end{tabular}

Fuente: Autores

\section{Conclusiones}

- Mediante la implementación de algoritmos y técnicas de procesamiento de imágenes se elaboró una aplicación grafica de fácil uso e interpretación de datos, que permite el procesamiento de imágenes satelitales mediante la modificación de parámetros (umbral, filtros espaciales) para discriminar e identificar formas y áreas de interés que contienen recursos hídricos. 
- Se determinó que la técnica de procesamiento visual de imágenes basadas en la selección manual del umbral genera mejores resultados, pues logra delimitar el área de estudio de mejor manera eliminando en su mayoría pixeles aislados, es decir, pixeles que no forman parte de los recursos hídricos encontrados, dejando de esa manera solo los pixeles que corresponden al elemento en análisis, por lo cual el cálculo del área es específico al área de estudio.

- El método automático de umbralización posee un funcionamiento óptimo en el cálculo del valor de umbralización, pero no es ideal para la aplicación desarrollada, pues este método considera pixeles aislados que según criterio de los observadores no forman parte de las imágenes en estudio, lo que altera de forma considerable en el cálculo del área del elemento analizado.

- La variación en la diferencia del área calculada en la aplicación se evidencia por la cantidad de pixeles que conforman las imágenes procesadas. Estadísticamente se empleó la prueba T-Student para medidas de dos muestras emparejadas considerando un nivel de confianza del 5\%, al aplicar la prueba se obtuvo una probabilidad 0,0059 que es menor al nivel de significancia, lo que nos permite concluir las medias de las áreas obtenidas por los dos métodos son diferentes. Por, los resultados estadísticos se concluyen que el método de umbralización manual es el más eficiente.

- Según el criterio del equipo, se desarrolla un mejor procesamiento digital de imágenes satelitales mediante la umbralización manual, pues según el criterio de los usuarios del sistema se pueden aumentar o disminuir la cantidad de información presente en la captura satelital, de esa manera, se puede seccionar de mejor forma el elemento en estudio.

\section{Referencias Bibliográficas}

Affairs, T. U. (12 de Noviembre de 2016). (UNOOSA. Obtenido de Access to Space4All: http://www.unoosa.org/oosa/index.html

Andy. (Agosto de 2018). Pixalytics. Obtenido de How many satellites are orbiting the Earth in 2018?: https://www.pixalytics.com/sats-orbiting-the-earth-2018/

Barragán, D. (15 de Enero de 2017). Manual de Interfaz Gráfica de Usuario en Matlab. Obtenido de Matlab: 
https://www.dspace.espol.edu.ec/bitstream/123456789/10740/19/\%255Bmatlab\%255D_ MATLAB_GUIDE.pdf

Copernicus, T. (2018). Europe's eyes of Earth. Obtenido de Copernicus: https://www.copernicus.eu/en

Díaz, I. (2016). Memorias de la Décima Quinta Conferencia Iberoamericana en Sistemas, Cibernética e Informática (CISCI 2016). Obtenido de Selección Automática de Umbrales para la Segmentación de Tumores Cerebrales en: http://www.iiis.org/CDs2016/CD2016Summer/papers/CA040RP.pdf

EESA. (Septiembre de 2019). Copernicus. Obtenido de Observing the Earth: http://www.esa.int/Our_Activities/Observing_the_Earth/Copernicus

EUMETSAT. (12 de Marzo de 2018). Obtenido de COPERNICUS ONLINE DATA ACCESS: https://www.eumetsat.int/website/home/Data/DataDelivery/CopernicusOnlineDataAcces s/index.html

Fresno, M. d. (2012). UN MÉTODO DE SEGMENTACIÓN DE IMÁGENES DIGITALES. Obtenido de UN MÉTODO DE SEGMENTACIÓN DE IMÁGENES DIGITALES: https://pdfs.semanticscholar.org/10bd/44ffd42b93b7757ad05c35588e8598cf5633.pdf

García, I. (2018). SCRIBD. Obtenido de Vision Artificial y Procesamiento Digital de Imagenes Usando Matlab.

González, F. (Enero de 2017). Tutorial de Teledeteccion Espacial. Obtenido de TELECAN: https://www.grss-ieee.org/wp-content/uploads/2014/07/ES_TUTORIAL_COMPLETO.pdf

Madrid, U. P. (Junio de 2015). Prácticas de Segmentación de las imágenes. Obtenido de DEPARTAMENTO DE ELECTRÓNICA,: http://www.elai.upm.es/webantigua/spain/Asignaturas/Robotica/PracticasROVA/prROVA 5Segmentacion.pdf

Manna, A. (Abril de 2016). Introduccion al Procesamientode Imagenes con Matlab 1 parte. Obtenido de Introduccion al Procesamientode Imagenes con Matlab: https://es.slideshare.net/FLliuya/introduccion-al-procesamiento-de-imagenes-con-matlab

Nations, U. (17 de Mayo de 2018). Office for Outer Space Affairs. Obtenido de Report of the InterAgency Meeting on Outer Space Activities (UN-Space) on its thirty-seventh session: http://www.unoosa.org/oosa/oosadoc/data/documents/2018/aac.105/aac.1051143_0.ht $\mathrm{ml}$

Ruescas, A. (Enero de 2009). Research Gate. Obtenido de La formación del campo térmico marino en la cuenca occidental del Mediterráneo: https://www.researchgate.net/publication/241775231_La_formacion_del_campo_termic o_marino_en_la_cuenca_occidental_del_Mediterraneo 
Unipython. (5 de Abril de 2018). Umbralización Adaptativa. Obtenido de UMBRALIZACIÓN DE UNA IMAGEN: https://unipython.com/umbralizacion-una-imagen/

Vara, F. (2016). Enseñando Optica Coherente usando Matlab GUIDE. Obtenido de Universidad Politecnica de Valencia.:

https://www.researchgate.net/publication/305026943_Ensenando_Optica_Coherente_us ando_Matlab_GUIDE

\section{PARA CITAR EL ARTÍCULO INDEXADO.}

Tinajero León, J., Lozada Yánez, P., Zabala Haro, M., \& Jiménez Granizo, C. (2019). Sistema de Procesamiento Digital de Imágenes Satelitales para Cálculo de Áreas de Interés. Ciencia Digital, 3(3.4.), 29-48. https://doi.org/10.33262/cienciadigital.v3i3.4.832

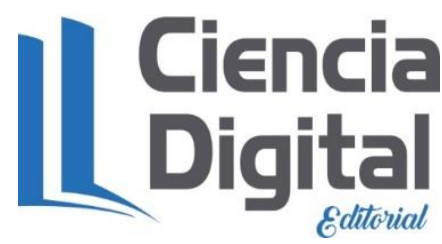

El artículo que se publica es de exclusiva responsabilidad de los autores y no necesariamente reflejan el pensamiento de la Revista Ciencia Digital.

El artículo queda en propiedad de la revista y, por tanto, su publicación parcial y/o total en otro medio tiene que ser autorizado por el director de la Revista Ciencia Digital.
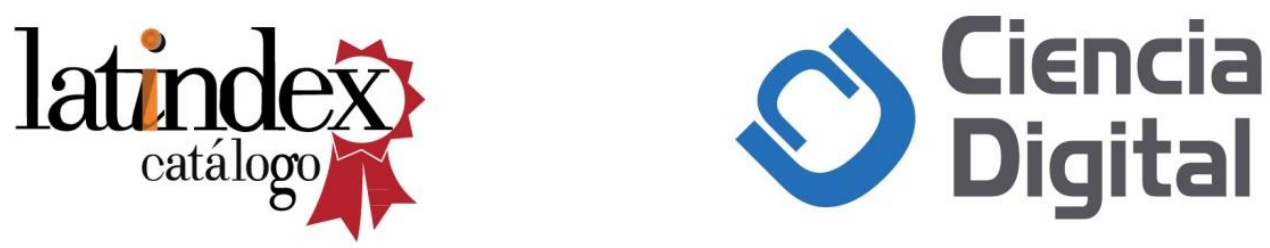